\title{
Erratum to: Direct Synthetic Processes for Cyclic Carbonates from Olefins and $\mathrm{CO}_{2}$
}

\author{
Jingsong Sun $\cdot$ Lin Liang $\cdot$ Jianmin Sun $\cdot$ \\ Yanqiu Jiang $\cdot$ Kaifeng Lin $\cdot$ Xianzhu Xu \\ Runwei Wang
}

Published online: 11 January 2011

(C) Springer Science+Business Media, LLC 2011

\section{Erratum to: Catal Surv Asia \\ DOI 10.1007/s10563-010-9106-4}

The original version of this article unfortunately contained a mistake. The affiliation "Harbin Institute of Technology University" of the authors Lin Liang, Jianmin Sun, Yanqiu
Jiang, Kaifeng Lin, Xianzhu Xu should read as "Harbin Institute of Technology".

The authors regret the error and would like to apologize for any inconvenience this may have caused to the readers of the journal.

The online version of the original article can be found under doi:10.1007/s10563-010-9106-4.

L. Liang · J. Sun $(\bowtie) \cdot$ Y. Jiang $\cdot$ K. Lin $\cdot$ X. Xu

State Key Laboratory of Urban Water Resource

and Environment, Harbin Institute of Technology,

Harbin 150090, China

e-mail: sunjm@hit.edu.cn

J. Sun $\cdot$ Y. Jiang $\cdot$ K. Lin $\cdot$ X. Xu

Natural Science Research Center, The Academy of Fundamental and Interdisciplinary Science, Harbin Institute of Technology,

Harbin 150080, China

J. Sun

Beijing University of Chemical Technology, Beijing, China

R. Wang

State Key Laboratory of Inorganic Synthesis and Preparative

Chemistry, College of Chemistry, Jilin University,

Changchun 130012, People's Republic of China 